\title{
Sources of Resistance for Charcoal Rot of Sorghum Caused by Macrophomina phaseolina (Tassi) Goid.
}

\author{
Vinayaka A. Bannur $^{2 *}$ and S. N. Chattannavar ${ }^{1}$ \\ ${ }^{1}$ All India Coordinated Sorghum Improvement Project, ${ }^{2}$ Department of Plant Pathology, \\ College of Agriculture Dharwad, University of Agricultural Sciences, \\ Dharwad - 580005, India \\ *Corresponding author
}

Keywords

Sorghum, Charcoal rot, Macrophomina phaseolina

Article Info

Accepted:

18 November 2019

Available Online:

10 December 2019

\section{A B S T R A C T}

Seventy-eight genotypes were screened for charcoal rot resistance in the sick plot during rabi 2018-19. The results of the study indicated that the genotypes E 36-1 (11.53 \%), followed by DSV 4 (12.24 \%), Dagadi Solapur $(13.40 \%)$ showed less disease incidence compared to other genotypes. Highest per cent charcoal rot incidence was recorded in CSV 8R $(54.34 \%)$. The study also revealed that the local genotypes recorded reduced levels of other charcoal rot parameters such as per cent lodging due to charcoal rot (soft stalk), mean length of spread (MLS) and mean number of nodes crossed (MNC). The local genotypes also possessed desired breeding traits such as delayed senescence and stay green type which could be employed in resistance breeding programme of rabi sorghum.

\section{Introduction}

Sorghum [Sorghum bicolor (Linn.) Moench] has occupied an area of 56 lakh hectares with the production of 46 lakh tons and productivity of $812 \mathrm{~kg} / \mathrm{ha}$. Themajor sorghum cultivating states are Maharashtra, Karnataka, Rajasthan, Tamil Nadu and Andhra Pradesh. It is being grown in two seasons: kharif season as a rainfed crop while in rabi season under remaining soil moisture conditions. In Karnataka, it is cultivated on 10.90 lakh hectares of which 1.16 lakh hectares in kharif and 9.74 lakh hectares in rabi with production and productivity of 11.50 lakh tons and 1,052 $\mathrm{kg} \mathrm{ha}^{-1}$ respectively (Anon., 2017).

Charcoal rot caused by Macrophomina phaseolina (Tassi) Goid. is major disease among biotic stresses in post rainy season and it cause major losses in grain and fodder yield. In India, almost all the cultivated hybrids and varieties are susceptible to charcoal rot (Jahagirdar, 2007). The indirect loss computed 
due to this disease alone amounts to 40 per cent (Hiremath and Palakshappa, 1994). Patil (1980) reported that loss in grain yield was more in rabi $(40.83 \%)$ than in kharif (17.69 $\%)$.With this background, present investigation was made to screen seventyeight genotypes for charcoal rot resistance.

\section{Materials and Methods}

Afield experiment was conducted at Main Agricultural Research Station, Dharwad in sick plot conditions during rabi 2018-19. Test genotypes were sown during the second fortnight of October with a spacing of $45 \mathrm{~cm} \times 15 \mathrm{~cm}$ with three replications. The susceptible check, CSV8R was sown after two test entries. Observations on charcoal rot incidence, mean length of spread $(\mathrm{cm})$, mean number of nodes crossed and charcoal rot index (CRI) were recorded for screening purpose. Charcoal rot percentage and mean length of spread of lesion were used for estimation of charcoal rot index (CRI) using the formula $(\mathrm{CRI}=\mathrm{CRP} \times 0.4+\mathrm{MLS} \times$ 0.6).Disease reaction of each genotype was determined following the CRI scales (Das et al., 2018).

\begin{tabular}{|l|l|}
\hline CRI VALUE & Disease Reaction \\
\hline$\leq 5$ & Highly Resistant \\
\hline $6-10$ & Resistant \\
\hline $11-25$ & Moderately resistant \\
\hline $26-40$ & Susceptible \\
\hline$>40$ & Highly susceptible \\
\hline
\end{tabular}

\section{Results and Discussion}

The results revealed that, per cent charcoal rot was least in E 36-1 (11.53 \%) followed by DSV 4 (12.24 \%) and Dagadi Solapur (13.40 $\%)$. Highest per cent charcoal rot incidence recorded in CSV 8R(54.34 \%). Least mean number of nodes crossed was recorded in $\mathrm{E}$ 36-1 (1.50), Kadumcuky (1.50) followed by DSV 4 (1.60) and Solapur Dagadi (1.60).
Highest mean nodes crossed was recorded in CSV 8R (3.00). The least mean length of spread was noticed in E $36-1(7.70 \mathrm{~cm})$ followed by DSV $4(12.33 \mathrm{~cm})$. The highest mean length of infection was recorded in CSV $8 \mathrm{R}(35.40 \mathrm{~cm})$. In case of charcoal rot index (CRI), the lowest CRI was recorded in E 36-1 (9.23) followed by DSV 4 (12.30) and Dagadi Solapur (16.61). The highest CRI value was recorded in CSV 8R (43.00) (Table 1).

Out of seventy-eight genotypes screened against charcoal rot incidence in sick plot, none of the genotype showed highly resistant reaction, only one genotype i.e., E 36-1 showed resistant reaction. Nineteen genotypes showed moderately resistant reaction. Fiftyseven genotypes showed susceptible disease reaction. One genotype i.e., CSV 8R showed highly susceptible reaction (Table 2 ).

The results of the screening were in accordance with studies made by Virupaksha Prabhu et al., (2012) who reported that the genotypes Dagadi Solapur (12.35\%) followed by GRS 1 (13.15\%) and BCR 9 (14.25\%) showed less charcoal rot incidence compared to other genotypes. CSV 8R genotype recorded higher disease incidence $(56.10 \%)$. Anahosur and Naik (1985) reported that susceptible genotypes possess less sugar content compared to resistant genotypes. Similarly, Nalawade et al., (2008) reported that resistant genotypes have more amount of sugar and phenolic compounds which confer resistant against the pathogen. In this present study, this may be the reason for genotypes showing resistant and susceptible disease reaction. Thus, from the results it is clear that employment of newer resistance sources particularly E 36-1 and Dagadi Solapur have shown resistance to charcoal rot over the years and can be effectively employed in resistance breeding programme against charcoal rot in sorghum. 
Table.1 Field evaluation of sorghum genotypes against charcoal rot of sorghum

\begin{tabular}{|c|c|c|c|c|c|}
\hline $\begin{array}{l}\text { Sl. } \\
\text { No. }\end{array}$ & Genotype name & $\begin{array}{c}\text { Charcoal } \\
\text { rot } \\
\text { incidence } \\
(\%)\end{array}$ & $\begin{array}{c}\text { Mean nodes } \\
\text { crossed } \\
\text { (No.) }\end{array}$ & $\begin{array}{c}\text { Mean } \\
\text { length of } \\
\text { spread }(\mathrm{cm})\end{array}$ & $\begin{array}{l}\text { Charcoal } \\
\text { rot index }\end{array}$ \\
\hline 1. & KaradaMaldandi & 38.88 & 2.25 & 26.60 & 31.80 \\
\hline 2. & Kekri Local & 24.24 & 2.00 & 28.00 & 26.50 \\
\hline 3. & YermalDakuri & 29.72 & 2.00 & 26.00 & 27.50 \\
\hline 4. & E 36-1 & 11.53 & 1.50 & 07.70 & 09.23 \\
\hline 5. & Pop Sorghum & 38.23 & 2.40 & 25.00 & 30.30 \\
\hline 6. & Tandalw & 35.29 & 1.66 & 25.33 & 29.31 \\
\hline 7. & IS 14338 & 46.42 & 2.00 & 27.50 & 35.07 \\
\hline 8. & M 35-1 & 35.13 & 2.40 & 28.00 & 30.85 \\
\hline 9. & Bulk Y & 20.50 & 2.20 & 21.00 & 20.80 \\
\hline 10. & IS 8283 & 37.83 & 2.00 & 28.75 & 32.38 \\
\hline 11. & IS 3546 & 37.50 & 2.25 & 28.00 & 31.93 \\
\hline 12. & SPV 1829 & 33.33 & 2.00 & 25.66 & 28.75 \\
\hline 13. & Solapur M 35-1 & 32.50 & 2.50 & 28.25 & 29.95 \\
\hline 14. & Phule Anuradha & 28.12 & 2.00 & 28.50 & 28.34 \\
\hline 15. & DSV 4 & 12.24 & 1.60 & 12.33 & 12.30 \\
\hline 16. & CSV 8R & 54.34 & 3.00 & 35.40 & 43.00 \\
\hline 17. & CSV 22 & 35.89 & 2.00 & 27.50 & 30.85 \\
\hline 18. & Parbhani Moti & 36.36 & 2.00 & 25.33 & 29.74 \\
\hline 19. & SPV 2217 & 22.22 & 2.00 & 23.50 & 23.00 \\
\hline 20. & Phule Panchami & 39.53 & 2.00 & 28.00 & 32.61 \\
\hline 21. & IS 8607 & 33.33 & 2.20 & 29.00 & 29.35 \\
\hline 22. & PVK Kranti & 29.54 & 2.40 & 26.70 & 27.83 \\
\hline 23. & Dagadi Solapur & 13.04 & 1.60 & 19.00 & 16.61 \\
\hline 24. & M 35-1 Akola & 34.61 & 2.40 & 28.76 & 31.1 \\
\hline 25. & Phule Vasudha & 34.14 & 2.40 & 27.25 & 30.00 \\
\hline 26. & JalnaDagdi & 26.19 & 2.25 & 24.00 & 24.87 \\
\hline 27. & SGMRN1-3-2 & 23.72 & 2.00 & 28.00 & 26.28 \\
\hline 28. & Phule Maubee & 27.27 & 2.40 & 26.25 & 26.65 \\
\hline
\end{tabular}


Contd......

\begin{tabular}{|c|c|c|c|c|c|}
\hline $\begin{array}{l}\text { Sl. } \\
\text { No. }\end{array}$ & Genotype name & $\begin{array}{l}\text { Charcoal rot } \\
\text { incidence (\%) }\end{array}$ & $\begin{array}{l}\text { Mean nodes } \\
\text { crossed (No.) }\end{array}$ & $\begin{array}{c}\text { Mean length } \\
\text { of spread (cm) }\end{array}$ & $\begin{array}{l}\text { Charcoal } \\
\text { rot index }\end{array}$ \\
\hline 29. & Annigeri & 25.53 & 2.00 & 22.33 & 23.61 \\
\hline 30. & Muguti Dharwad & 24.39 & 2.00 & 23.70 & 23.97 \\
\hline 31. & Kadumcuky & 36.00 & 1.50 & 23.00 & 28.20 \\
\hline 32. & Phule Chitra & 29.26 & 2.00 & 20.50 & 24.00 \\
\hline 33. & Barsi Jowar & 34.09 & 2.00 & 27.70 & 30.25 \\
\hline 34. & BJV 44 & 19.56 & 1.67 & 20.70 & 20.24 \\
\hline 35. & M 35-1-1 & 33.53 & 2.00 & 26.50 & 29.23 \\
\hline 36. & M 35-1-2 & 36.53 & 2.00 & 27.80 & 31.30 \\
\hline 37. & BarsiJoot & 30.30 & 2.00 & 25.33 & 27.31 \\
\hline 38. & Tandur Local & 26.08 & 2.20 & 21.70 & 23.45 \\
\hline 39. & Harini Jagadi & 32.60 & 2.20 & 23.25 & 27.00 \\
\hline 40. & E 24 Kdagdi & 38.46 & 2.00 & 22.00 & 28.58 \\
\hline 41. & Kddgn Local & 25.58 & 2.00 & 27.20 & 26.55 \\
\hline 42. & Biligunda & 28.84 & 2.60 & 23.50 & 25.63 \\
\hline 43. & Karjat Local & 26.67 & 2.00 & 27.50 & 27.16 \\
\hline 44. & Tassal & 24.39 & 2.20 & 22.33 & 23.15 \\
\hline 45. & Kadbina Jola & 20.51 & 2.40 & 27.00 & 24.40 \\
\hline 46. & Yarnal Local & 39.47 & 2.20 & 22.70 & 29.40 \\
\hline 47. & ChungiMaldandi & 32.43 & 2.00 & 27.70 & 29.59 \\
\hline 48. & Maldandi Local & 34.21 & 2.00 & 28.70 & 30.90 \\
\hline 49. & Yerimala Local & 38.89 & 2.20 & 27.80 & 32.23 \\
\hline 50. & TangriniganMaldandi 3 & 31.81 & 2.25 & 27.33 & 29.12 \\
\hline 51. & SikkandarLakkadi & 26.47 & 2.30 & 27.00 & 26.78 \\
\hline 52. & Kodaimky & 36.58 & 2.00 & 26.50 & 30.53 \\
\hline 53. & DSV 5 & 25.58 & 2.40 & 21.75 & 23.28 \\
\hline 54. & Phule Revati & 24.39 & 2.40 & 27.00 & 25.95 \\
\hline 55. & Chappalgaon Local & 35.89 & 2.00 & 28.00 & 31.15 \\
\hline 56. & Mssbr Local & 32.65 & 2.00 & 26.33 & 28.85 \\
\hline 57. & В 58586 & 27.27 & 2.00 & 21.00 & 23.50 \\
\hline 58. & Hardakan Local & 33.33 & 2.50 & 28.00 & 30.13 \\
\hline
\end{tabular}


Contd......

\begin{tabular}{|c|c|c|c|c|c|}
\hline $\begin{array}{l}\text { Sl. } \\
\text { No. }\end{array}$ & Genotype name & $\begin{array}{l}\text { Charcoal rot } \\
\text { incidence }(\%)\end{array}$ & $\begin{array}{c}\text { Mean nodes } \\
\text { crossed } \\
(\text { No. })\end{array}$ & $\begin{array}{c}\text { Mean } \\
\text { length of } \\
\text { spread }(\mathrm{cm})\end{array}$ & $\begin{array}{l}\text { Charcoal } \\
\text { rot index }\end{array}$ \\
\hline 59. & IS 14332 & 28.57 & 2.33 & 28.25 & 28.37 \\
\hline 60. & IS 27042 & 22.50 & 2.25 & 28.00 & 25.80 \\
\hline 61. & H 112 & 33.33 & 2.00 & 23.70 & 27.55 \\
\hline 62. & IS $8757-2-3$ & 20.83 & 2.25 & 28.00 & 25.13 \\
\hline 63. & IS 8185 & 25.53 & 2.75 & 29.00 & 27.61 \\
\hline 64. & IS 3547 & 40.00 & 2.40 & 25.70 & 31.42 \\
\hline 65. & CSV 216R & 25.00 & 2.60 & 27.50 & 26.50 \\
\hline 66. & Maldandi Local & 29.54 & 2.40 & 28.00 & 28.61 \\
\hline 67. & SPV 1231 & 25.00 & 2.20 & 30.00 & 28.00 \\
\hline 68. & SPV 475 & 32.00 & 2.20 & 27.33 & 29.19 \\
\hline 69. & DSFR 5 & 28.94 & 2.00 & 26.33 & 27.37 \\
\hline 70. & AKFS 3 & 29.03 & 2.00 & 28.70 & 28.83 \\
\hline 71. & DMR 1 & 26.31 & 2.00 & 22.33 & 23.92 \\
\hline 72. & DBR 1 & 32.50 & 2.00 & 26.70 & 29.02 \\
\hline 73. & IS 2205 & 23.68 & 2.25 & 28.00 & 26.27 \\
\hline 74. & IS 2312 & 29.41 & 2.00 & 28.25 & 28.71 \\
\hline 75. & DMR 2 & 27.50 & 1.75 & 22.70 & 24.62 \\
\hline 76. & IS 18551 & 36.80 & 2.25 & 28.25 & 31.67 \\
\hline 77. & DSV 3 & 21.42 & 2.00 & 21.75 & 21.61 \\
\hline 78. & DSFR 1 & 34.21 & 2.00 & 24.25 & 28.23 \\
\hline
\end{tabular}


Table.2 Reaction of sorghum genotypes to charcoal rot

\begin{tabular}{|c|c|c|}
\hline CRI value & Genotypes & Disease reaction \\
\hline$<5$ & Nil & Highly resistant \\
\hline 5-10 & E $36-1$ & Resistant \\
\hline 11-25 & $\begin{array}{l}\text { DSV 4, SPV 2217, Dagadi Solapur, JalnaDagdi, Annigeri, Muguti Dharwad, Phule Chitra, } \\
\text { BJV 44, Tandur Local, Biligunda, Tassal, Kadbina Jola, DSV 5, B 58586, IS 8757-2-3, DMR } \\
\text { 1, DMR 2, DSV 3, Bulk Y }\end{array}$ & Moderately resistant \\
\hline 26-40 & $\begin{array}{l}\text { KaradaMaldandi, Kekri Local, YermalDakuri, Pop Sorghum, Tandalw, IS 14338, M 35-1, IS } \\
\text { 8283, IS 3546, SPV 1829, Solapur M 35-1, Phule Anuradha, CSV 22, Parbhani Moti, Phule } \\
\text { Panchami, IS 8607, PVK Kranti, M 35-1 Akola, Phule Vasudha, SGMRN1-3-2, Phule } \\
\text { Maubee, Kadumcuky, Barsi jowar, M 35-1-1, M 35-1-2, Barsijoot, Harini Jagadi, E } 24 \\
\text { Kdagdi, Kddgn Local, Kajarat Local, Yarnal Local, ChungiMaldandi, Maldandi Local, } \\
\text { Yerimala Local, TengriniganMaldnadi 3, Kodaimky, Phule Revati, Chappalgaon Local, } \\
\text { Mssbr Local, Hardakan Local, IS 14332, IS 27042, H 112, IS 8185, IS 3547, CSV 216R, } \\
\text { MaldandiLakkadi, SPV 1231, SPV 475, DSFR 5, AKFS 3, DMR 1, DBR 1, IS 2205, IS 2312, } \\
\text { IS 18551, DSFR } 1\end{array}$ & Susceptible \\
\hline$>40$ & CSV 8R & Highly susceptible \\
\hline
\end{tabular}




\section{References}

Anahosur, K. H. and Naik, S. T., 1985, Relationships of sugar and phenols of root and stalk rot of sorghum with charcoal rot. Indian Phytopath., 38 (1): 131-134.

Anonymous., 2017, Selected state/seasonwise area, production and productivity of jowar in India. Ministry of Agriculture and Farmers' Welfare, Govt. of India, New Delhi, pp. 97-99.

Arora, M. and Dhurwe, U., 2014, Grain yield losses due to charcoal rot of sorghum infected by Macrophomina phaseolina. Global. J. Biol. Agri. Health. Sci., 3 (1): 267-269.

Das, I. K., Rakshit, S., Sharma, K. K., Chattannavar, S. N., Gholve, V. M., Jayalakshmi, S. K. and Tonapi, V. A., 2018, Development of a charcoal rot index for multilocation trials of sorghum. Crop Prot., 108: 102-109.

Hiremath, R. V. and Palakshappa, M. G., 1994, Severe incidence of charcoal rot of sorghum at Dharwad. Curr. Sci., 33: 44.

Jahagirdar, S., 2007, Present status and future research needs on the management of charcoal rot of sorghum. Agric. Rev., 28(3): 197-206.

Nalawade, S. V., Agarkar, G. D. and Chirame, B. B., 2008, Biochemical mechanism of host resistance to Macrophomina phaseolina (Tassi) Goid. of sorghum. J. Maharashtra Agric. Univ., 33(2): 193-195.

Patil, S. H., 1980, Studies on charcoal rot of sorghum caused by Macrophomina phaseolina (Tassi) Goid. M. Sc. (Agri.) Thesis, Univ. of Agric. Sci., Bangalore, Karnataka (India).

Virupaksha Prabhu, H., Adiver, S. S., and Basavarajappa, M. P., 2012, Field screening of germplasm lines and local genotypes against charcoal rot of sorghum caused by Macrophomina phaseolina (Tassi) Goid. Int. J. Plant Prot., 2 (5): 299-303.

\section{How to cite this article:}

Vinayaka A. Bannur and Chattannavar, S. N. 2019. Sources of Resistance for Charcoal Rot of Sorghum Caused by Macrophomina phaseolina (Tassi) Goid. Int.J.Curr.Microbiol.App.Sci. 8(12): 2539-2545. doi: https://doi.org/10.20546/ijcmas.2019.812.297 